\title{
Improving Transmission by MIMO Channel Structuring
}

\author{
Markus Rupp and Christoph F. Mecklenbräuker
}

\begin{abstract}
In this paper, new space-time coding schemes for multiple transmit and receive antenna systems (MIMO) are proposed. By applying a specific block coding scheme for transmission, the MIMO channel is structured in an advantageous manner. Diversity can be gained simultaneously with savings in receiver complexity. In a second step, such structured schemes and BLAST schemes are combined, offering a continuous trade-off between Quality of Service (QoS) and choice of data rate. Due to the simplicity of the coding schemes, only very moderate modifications in the existing UMTS standard are required in order to support them. If the transmitter knows the numbers of antenna elements at both sides of the radio link then this is sufficient for selecting the most appropriate scheme.
\end{abstract}

\section{INTRODUCTION}

In the following, the well known scheme of Alamouti[1], [2] for two transmit antennas is revisited. An extension to a four transmit antenna scheme (and in general $M=2^{m}$ transmit antennas) is presented in Section II. A detailed description can be found in [3]. Section III further extensions are proposed allowing 1) arbitrary numbers of receive antennas and 2) a generalization, called Alamoutization is shown for multiple transmit and receive antennas not being of order $2^{m}$ but of arbitrary even number. Such procedures allow structuring of an otherwise unstructured wireless channel and thus reduction in complexity at the receiver end as well as preserving diversity gain for transmission. In Section IV it will be demonstrated that such flexible structuring can lead to optimal usage of resources, namely the wireless channel. By reducing the block code length less diversity is achieved, while the complexity suffers only moderately. This allows transmitting data at a higher rate when sufficient channel quality (in terms of diversity) is available and reducing the rate otherwise in order to achieve higher diversity gain.

\section{Alamouti Scheme}

A very simple but effective scheme for two antennas achieving a diversity gain of two was proposed by Alamouti [1], [2]. It works by sending the sequence $\left\{s_{1}, s_{2}^{*}\right\}$ on the first antenna and $\left\{s_{2},-s_{1}^{*}\right\}$ on the other. Assuming a flatfading channel and denoting the two channel coefficients $h_{1}$ and $h_{2}$, the received vector $\mathbf{r}$ is formed by stacking two consecutive data samples $\left[r_{1}, r_{2}\right]^{T}$ in time

$$
\mathbf{r}=\mathbf{S h}+\overline{\mathbf{v}}
$$

M. Rupp is a Professor at TU Wien, Institute for Communication and RF Engineering, Gusshausstr. 25/389, A-1040 Vienna, Austria (email: mrupp@nt.tuwien.ac.at)

C.F. Mecklenbräuker is a key researcher at ftw., Vienna, DonauCity Str. 1, A-1220 Vienna, Austria (email: cfm@ftw.at)
Here, the symbol block $\mathbf{S}$ and the channel vector $\mathbf{h}$ are introduced,

$$
\mathbf{S}=\left[\begin{array}{rr}
s_{1} & s_{2} \\
s_{2}^{*} & -s_{1}^{*}
\end{array}\right], \quad \mathbf{h}=\left[\begin{array}{l}
h_{1} \\
h_{2}
\end{array}\right]
$$

This can be reformulated as

$$
\left[\begin{array}{l}
r_{1} \\
r_{2}^{*}
\end{array}\right]=\left[\begin{array}{rr}
h_{1} & h_{2} \\
-h_{2}^{*} & h_{1}^{*}
\end{array}\right]\left[\begin{array}{l}
s_{1} \\
s_{2}
\end{array}\right]+\left[\begin{array}{l}
v_{1} \\
v_{2}^{*}
\end{array}\right]
$$

or in short notation

$$
\mathbf{y}=\mathbf{H} \mathbf{s}+\mathbf{v}
$$

where $\mathbf{y}=\left[r_{1}, r_{2}^{*}\right]^{T}$. The resulting channel matrix $\mathbf{H}$ is orthogonal, i.e.

$$
\mathbf{H}^{H} \mathbf{H}=\mathbf{H} \mathbf{H}^{H}=h^{2} \mathbf{I}_{2},
$$

where the $2 \times 2$ identity matrix $\mathbf{I}_{2}$ as well as the gain of the channel $h^{2}=\left|h_{1}\right|^{2}+\left|h_{2}\right|^{2}$ was introduced, indicating a two times diversity for the reception of both symbols. Note that each matrix element $h_{i}$ is assumed to be a complex gaussian random variable with unit variance. The pdf of $h^{2}$ is a $\chi^{2}$ density function with $N$ degrees of diversity ( $2 N$ degrees of freedom). In this case two matrix elements were combined, thus the diversity order equals $N=2$. The transmitted symbols can be computed by the ZF approach,

$$
\hat{\mathbf{s}}=\left[\mathbf{H}^{H} \mathbf{H}\right]^{-1} \mathbf{H}^{H} \mathbf{y}=\frac{1}{h^{2}} \mathbf{H}^{H} \mathbf{y},
$$

which gives equivalent results to the ML solution due to the diagonal structure of the $\mathbf{H} \mathbf{H}^{H}$, however, with less receiver complexity. This can be further elaborated on:

$$
\hat{\mathbf{s}}=\mathbf{s}+\left[\mathbf{H}^{H} \mathbf{H}\right]^{-1} \mathbf{H}^{H} \mathbf{v}
$$

revealing the noise filtering. The noise variance for the two symbols is given by $\sigma_{\mathbf{v}}^{2} \operatorname{trace}\left(\left[\mathbf{H}^{H} \mathbf{H}\right]^{-1}\right)=2 \sigma_{\mathbf{v}}^{2} / h^{2}$. Using complex-valued modulation, only for the two antenna scheme such an improvement is possible. Only in the case of binary transmission, higher schemes with 4 , and 8 antennas exist [5]. In UMTS, QPSK is utilized on CDMA preventing perfectly orthogonal schemes with an improvement larger than a diversity of two.

In the following, a four times diversity scheme for UMTS is proposed. The scheme was already proposed in [6], but its full potential not recognized. It comes with a small caveat though which will be explained in the following. 
Proposition 2.1 Starting with the $2 \times 2$-Alamouti scheme the following recursive construction rule (similar to the construction of a Walsh-Hadamard code) is applied

$$
\left[\begin{array}{rr}
h_{1} & h_{2} \\
-h_{2}^{*} & h_{1}^{*}
\end{array}\right] \rightarrow\left[\begin{array}{rrrr}
h_{1} & h_{2} & h_{3} & h_{4} \\
-h_{2}^{*} & h_{1}^{*} & -h_{4}^{*} & h_{3}^{*} \\
-h_{3}^{*} & -h_{4}^{*} & h_{1}^{*} & h_{2}^{*} \\
h_{4} & -h_{3} & -h_{2} & h_{1}
\end{array}\right]
$$

i.e. the complex scalars $h_{1}$ and $h_{2}$ appearing to the left of the arrow " $\rightarrow$ " are replaced by the $2 \times 2$ matrices

$$
\mathbf{H}_{1}=\left[\begin{array}{rr}
h_{1} & h_{2} \\
-h_{2}^{*} & h_{1}^{*}
\end{array}\right] \quad \text { and } \quad \mathbf{H}_{2}=\left[\begin{array}{rr}
h_{3} & h_{4} \\
-h_{4}^{*} & h_{3}^{*}
\end{array}\right]
$$

and then re-inserted into the Alamouti Space-Time channel matrix

$$
\left[\begin{array}{rr}
\mathbf{H}_{1} & \mathbf{H}_{2} \\
-\mathbf{H}_{2}^{*} & \mathbf{H}_{1}^{*}
\end{array}\right]
$$

where ${ }^{*}$ denotes complex conjugation without transposition.

This results in the following symbol block $\mathbf{S}$ for transmitting the four symbols $\mathbf{s}=\left[s_{1}, \ldots, s_{4}\right]^{T}$ :

$$
\mathbf{S}=\left[\begin{array}{rrrr}
s_{1} & s_{2} & s_{3} & s_{4} \\
s_{2}^{*} & -s_{1}^{*} & s_{4}^{*} & -s_{3}^{*} \\
s_{3}^{*} & s_{4}^{*} & -s_{1}^{*} & -s_{2}^{*} \\
s_{4} & -s_{3} & -s_{2} & s_{1}
\end{array}\right]
$$

The received vector $\mathbf{r}$ can be expressed in the same form as (1). Converting the received vector by complex conjugation

$$
\begin{aligned}
& y_{1}=r_{1}, \quad v_{1}=\bar{v}_{1}, \\
& y_{2}=r_{2}^{*}, \quad v_{2}=\bar{v}_{2}^{*}, \\
& y_{3}=r_{3}^{*}, \quad v_{3}=\bar{v}_{3}^{*}, \\
& y_{4}=r_{4}, \quad v_{4}=\bar{v}_{4} .
\end{aligned}
$$

results in the following equivalent transmission scheme

$$
\mathbf{y}=\mathbf{H s}+\mathbf{v}
$$

in which $\mathbf{H}$ appears again as MIMO channel transmission matrix. If $\overline{\mathbf{v}}$ is a complex Gaussian vector with i.i.d. elements then so is $\mathbf{v}$. The resulting matrix $\mathbf{H}$ is not unitary anymore, instead

$$
\mathbf{H}^{H} \mathbf{H}=\mathbf{H H}^{H}=h^{2}\left[\begin{array}{rr}
\mathbf{I}_{2} & X \mathbf{J}_{2} \\
-X \mathbf{J}_{2} & \mathbf{I}_{2}
\end{array}\right]
$$

where the $2 \times 2$ matrix

$$
\mathbf{J}_{2}=\left[\begin{array}{rr}
0 & 1 \\
-1 & 0
\end{array}\right]
$$

has been introduced. The gain of the channel is

$$
h^{2}=\left|h_{1}\right|^{2}+\left|h_{2}\right|^{2}+\left|h_{3}\right|^{2}+\left|h_{4}\right|^{2},
$$

and the channel dependent real-valued random variable $X$ is defined by

$$
X=2\left[\operatorname{Real}\left(h_{1} h_{4}^{*}-h_{2} h_{3}^{*}\right)\right] / h^{2} .
$$

By applying the matched filter $\mathbf{H}^{H}$, this results in the reception of the following vector:

$$
\begin{aligned}
\mathbf{z} & =\mathbf{H}^{H} \mathbf{y}=\mathbf{H}^{H} \mathbf{H} \mathbf{s}+\mathbf{H}^{H} \mathbf{v} \\
& =h^{2}\left[\begin{array}{ccc}
s_{1} & + & X s_{4} \\
s_{2} & - & X s_{3} \\
s_{3} & - & X s_{2} \\
s_{4} & + & X s_{1}
\end{array}\right]+\mathbf{H}^{H} \mathbf{v}
\end{aligned}
$$

in which the pair $\left\{s_{1}, s_{4}\right\}$ is decoupled from $\left\{s_{2}, s_{3}\right\}$ allowing for a low complexity solution based on the newly formed receiver vector $\mathbf{z}$. Numerical results for the Bit Error Rate (BER) for variants of QPSK alphabets are presented and discussed in [3].

Although [7] shows that for general complex-valued random matrices $\mathbf{H}$, the diversity gain applying simple ZF schemes is only $M-N+1$, for such structured matrices it is different. The following solutions for ML and ZF are obtained:

$$
\begin{aligned}
\operatorname{BER}_{M L, A l} & =\frac{1}{2} \mathrm{E}_{h^{2}}\left[\operatorname{erfc}\left(\sqrt{\frac{h^{2}}{\sigma_{\mathbf{v}}^{2}}}\right)\right] \\
\mathrm{BER}_{Z F, A l} & =\frac{1}{2} \mathrm{E}_{h^{2}, X^{2}}\left[\operatorname{erfc}\left(\sqrt{\frac{\left(1-X^{2}\right) h^{2}}{\sigma_{\mathbf{v}}^{2}}}\right)\right]
\end{aligned}
$$

Remark on ZF: The term $\frac{\left(1-X^{2}\right) h^{2}}{\sigma_{\mathrm{v}}^{2}}$ showing in the ZF solution can be interpreted as a smaller diversity. $\mathrm{E}\left[X^{2}\right]=0.2$ and thus the diversity can be expected to be lowered from four to 3.2 .

Note that the ZF for such matrix $\mathbf{H}$ is substantially different than the general case. Thus, the conclusion from[7] that $=h^{2}\left(1-X^{2}\right)$ is $\chi^{2}$ distributed with one $(=4-4+1)$ degree of freedom is not correct. Figure1 displays a histogram of $\alpha$ from 3000 runs. Closest fit is the squared Nakagami-m distribution with $\mathrm{m}=3.2$, or equivalent $\chi^{2}$ with 6.4 degrees of freedom.

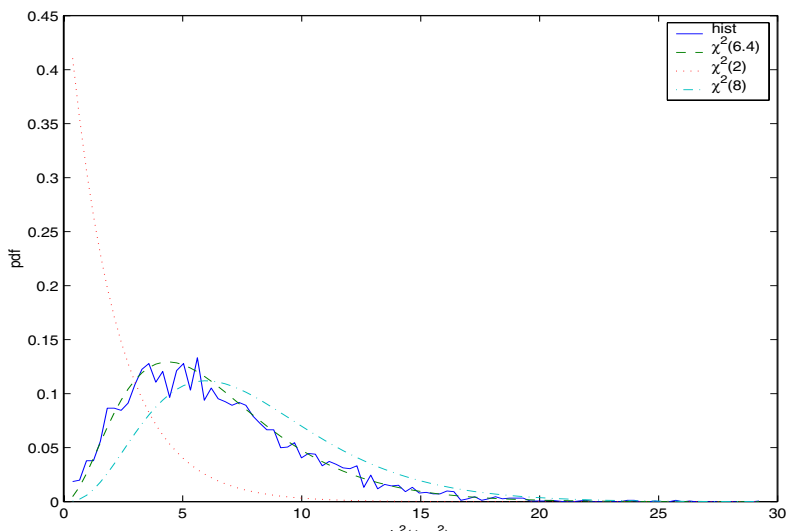

Fig. 1. PDF and histogram of $h^{h^{2}\left(1-x^{2}\right)}\left(1-X^{2}\right)$ for $4 x 1$ extended Alamouti scheme

Remark on ML: The maximum-likelihood receiver selects the $\mathbf{s}$ which minimizes

$$
\Lambda_{1}(\mathbf{s})=\|\mathbf{y}-\mathbf{H} \mathbf{s}\|^{2}=\mathbf{s}^{H} \mathbf{G s}-2 \operatorname{Re}\left(\mathbf{y}^{H} \mathbf{H} \mathbf{s}\right)+\|\mathbf{y}\| 2
$$


for all permissible symbol vectors $\mathbf{s}$ from the transmitter alphabet, introducing the Grammian

$$
\mathbf{G}=\mathbf{H}^{H} \mathbf{H} \text {. }
$$

The remainder of this section is specialized to QPSK which is relevant for UMTS. The transmitter alphabet of the four antenna scheme using QPSK consists of $4^{4}=256$ symbol vectors $\mathbf{s}$.

Alternatively, a matched filter can be applied to $\mathbf{y}$ and the maximum-likelihood estimator can be implemented on its output $\mathbf{z}=\mathbf{H}^{H} \mathbf{y}$ instead. However, it needs to be taken into account that the noise plus interference is spatially correlated after filtering: Assuming $\mathbf{v}$ to be zero-mean and covariance matrix $\sigma_{\mathbf{v}}^{2} \mathbf{I}_{4}$ gives a $\mathbf{w}=\mathbf{H}^{H} \mathbf{v}$ with conditional covariance matrix $\sigma_{\mathbf{v}}^{2} \mathbf{G}$ given the realization of $\mathbf{H}$.

The advantage of this approach is that this partly decouples the symbols. The pair $\left\{s_{1}, s_{4}\right\}$ is decoupled from $\left\{s_{2}, s_{3}\right\}$ allowing for a maximum-likelihood receiver which needs to search over 16 vector symbols only twice,

$$
\begin{aligned}
\Lambda_{2}(\mathbf{s}) & =(\mathbf{z}-\mathbf{G} \mathbf{s})^{H} \mathbf{G}^{-1}(\mathbf{z}-\mathbf{G} \mathbf{s}) \\
& =\Lambda_{2 a}\left(s_{1}, s_{4}\right)+\Lambda_{2 b}\left(s_{2}, s_{3}\right) .
\end{aligned}
$$

Maximization of $\Lambda_{2}(\mathbf{s})$ is achieved by individually maximizing $\Lambda_{2 a}\left(s_{1}, s_{4}\right)$ and $\Lambda_{2 b}\left(s_{2}, s_{3}\right)$ and we stress that maximizing $\Lambda_{2}(\mathbf{s})$ is strictly equivalent to maximizing $\Lambda_{1}(\mathbf{s})$.

\section{Alamoutisation}

So far only $M \times 1$ antenna schemes were considered. However, in near future more than one antenna will occur at the receivers as well. A cellular phone can carry two and a laptop even four antennas [8]. The proposed schemes could also applied, however, it remains unclear how to combine the received signals now in an optimal fashion.

A simple extension to the already proposed extended Alamouti scheme is the following. Assume $N$ antenna elements are available rather than one and that the spacing of the elements is sufficiently large so that the corresponding channel values $h_{n m}$ are all mutually uncorrelated. The received block vectors $\mathbf{y}_{1}, \mathbf{y}_{2}, \ldots, \mathbf{y}_{N}$ can be combined by

$$
\tilde{\mathbf{z}}=\sum_{n=1}^{N} \mathbf{H}_{n}^{H} \mathbf{y}_{n}
$$

The so obtained new vector is also given by

$$
\tilde{\mathbf{z}}=\sum_{n=1}^{N} \mathbf{H}_{n}^{H} \mathbf{H}_{n} \mathbf{s}+\sum_{n=1}^{N} \mathbf{H}_{n}^{H} \mathbf{v}_{n} .
$$

The new matrix $\tilde{\mathbf{G}}=\sum_{n=1}^{N} \mathbf{H}_{n}^{H} \mathbf{H}_{n}$ maintains its structure, thus can still be inverted by a simple $2 \times 2$ matrix inversion, preserving the structure of (10). Alternatively, the low complexity ML receiver can be used, since the two pairs $\left\{s_{1}, s_{4}\right\}$ and $\left\{s_{2}, s_{3}\right\}$ are still separable. The new value $h^{2}$ is given by

$$
h^{2}=\sum_{m=1}^{M} \sum_{n=1}^{N}\left|h_{n m}\right|^{2}
$$

indicating now the $N M$ times diversity.

While such scheme still requires the transmit antennas to be of order $2^{m}$, an interesting other approach is presented in the following allowing to increase the diversity when the number of receiver antennas is more than one but typically less than the number of transmit antennas. The proposed STC-schemes preserve as much orthogonality so that the receivers are of low complexity, while a high diversity is exploited.

Proposition 3.1 Assume that a block matrix form of the channel matrix $\mathbf{H}$ is given by

$$
\mathbf{H}=\left[\mathbf{H}_{1} \mathbf{H}_{2}\right],
$$

where the matrices $\left\{\mathbf{H} 1, \mathbf{H}_{2}\right\}$ are not necessarily quadratic. Then, the scheme can be Alamouted by performing the following operation:

$$
\mathbf{G}=\left[\begin{array}{rr}
\mathbf{H}_{1} & \mathbf{H}_{2} \\
-\mathbf{H}_{2}^{*} & \mathbf{H}_{1}^{*} \\
\mathbf{H}_{2}^{*} & \mathbf{H}_{1}^{*} \\
\mathbf{H}_{1} & -\mathbf{H}_{2}
\end{array}\right]
$$

At the receiver a $\mathrm{ZF}$ operation is performed, obtaining the corresponding term $\mathbf{G}^{H} \mathbf{G}$ with the property

$$
\mathbf{G}^{H} \mathbf{G}=2\left[\begin{array}{rr}
\mathbf{H}_{1}^{H} \mathbf{H}_{1}+\mathbf{H}_{2}^{H} \mathbf{H}_{2} & 0 \\
0 & \mathbf{H}_{1}^{H} \mathbf{H}_{1}+\mathbf{H}_{2}^{H} \mathbf{H}_{2}
\end{array}\right] .
$$

Thus, a perfect orthogonality on the non-diagonal block entries is achieved indicating low complexity at the receiver while the diagonal block terms indicate high diversity values. In a simpler form this was also proposed in [9].

Example 3.1: a two transmit-two receive antenna system is considered.

$$
\mathbf{H}_{1}=\left[\begin{array}{l}
h_{1} \\
h_{2}
\end{array}\right], \quad \mathbf{H}_{2}=\left[\begin{array}{l}
h_{3} \\
h_{4}
\end{array}\right]
$$

The matrix $\mathbf{G}^{H} \mathbf{G}$ becomes

$$
\mathbf{G}^{H} \mathbf{G}=2\left(\left|h_{1}\right|^{2}+\left|h_{2}\right|^{2}+\left|h_{3}\right|^{2}+\left|h_{4}\right|^{2}\right)\left[\begin{array}{ll}
1 & 0 \\
0 & 1
\end{array}\right]
$$

Thus, the full four times diversity can be explored, without a matrix inverse computation. Note that in this case, the sequence to transmit at the two antennas reads

$$
\left\{\begin{array}{rrrrrrrr}
s_{1} & s_{2} & -s_{3}^{*} & -s_{4}^{*} & s_{3}^{*} & s_{4}^{*} & s_{1} & s_{2} \\
s_{3} & s_{4} & s_{1}^{*} & s_{2}^{*} & s_{1}^{*} & s_{2}^{*} & -s_{3} & -s_{4}
\end{array}\right\}
$$

Note also that during eight time periods only four symbols are transmitted, i.e., this particular scheme has the drawback of offering only half the symbol rate!

Example 3.2 Consider a 4 by 2 transmission scheme. The matrices are identified to

$$
\mathbf{H}_{1}=\left[\begin{array}{ll}
h_{11} & h_{12} \\
h_{21} & h_{22}
\end{array}\right], \quad \mathbf{H}_{2}=\left[\begin{array}{ll}
h_{13} & h_{14} \\
h_{23} & h_{24}
\end{array}\right]
$$


The matrix $\mathbf{G}^{H} \mathbf{G}$ consists of two block matrices of size $2 \times 2$ on the diagonal. Thus, the scheme is still rather simple, since only a $2 \times 2$-matrix has to be inverted although a four-path diversity is achieved. A comparison of the noise enhancement shows that for this $4 \times 2$ antenna system $3 \mathrm{~dB}$ is gained compared to the $4 \times 1$ antenna system. Note that now the data rate is at full speed!

Example 3.3 The previously discussed $4 \times 1$ antenna system can be obtained when setting

$$
\mathbf{H}_{1}=\left[\begin{array}{ll}
h_{1} & h_{2}
\end{array}\right], \quad \mathbf{H}_{2}=\left[\begin{array}{ll}
h_{3} & h_{4}
\end{array}\right]
$$

The reader may also try schemes in which the number of receive antennas is not given by $N=2^{n}$. As long as $N$ is even, the scheme can be separated in two matrices $\mathbf{H}_{1}$ and $\mathbf{H}_{2}$ of same size allowing the Alamoutization rule 3.1.

\section{Combining BLAST and Alamouti Schemes}

Although the proposed extended Alamouti schemes allow for utilizing the channel diversity without sacrificing the receiver complexity, nothing has been said on data rates yet. In the case of $M \times 1$ antenna schemes, the $M$ symbols were repeated $M$ times in a different and specific order guaranteeing a data rate of one. Thus, the data rates in the proposed schemes typically remain constant (equal to one) when the schemes are quadratic and can be lower when the receive antenna number is smaller than the transmit antenna as pointed out in the previous section. In BLAST transmission this is different. In its simplest form, the V-BLAST coding [10], $M$ new symbols are offered to the $M$ transmit antennas at every symbol time instant thus achieving data rates $M$ times higher than in the Alamouti schemes. A combination of schemes can be achieved by simply transmitting more or less of the different repetitive transmissions. By utilizing the so obtained transmission matrix structures the diversity inherent in the transmission scheme can be exploited differently offering a trade-off between data-rate and diversity order. In order to clarify this statement, two example are presented.

Example 4.1 A $4 \times 2$ antenna scheme is considered for transmission. In a flat channel system, eight Rayleigh coefficients are available describing the transmissions from the four transmit to the two receive antennas, the transmission matrix being

$$
\mathbf{H}=\left[\begin{array}{llll}
h_{11} & h_{12} & h_{13} & h_{14} \\
h_{21} & h_{22} & h_{23} & h_{24}
\end{array}\right] .
$$

It should thus be possible to transmit either four times the symbol data rate with diversity gain two, or two times the data rate with diversity four or only at symbol data rate but with diversity gain eight. In the first case the $4 \times 4$ scheme as proposed in Section II will be used, repeating the four symbols four times, resulting in the reception of eight symbols. When assigning two paths each to one $2 \times 2$ matrix $\mathbf{H}_{i}, i=1 . .4$, the following transmission matrix is obtained

$$
\mathbf{H}=\left[\begin{array}{rr}
\mathbf{H}_{1} & \mathbf{H}_{2} \\
-\mathbf{H}_{2}^{*} & \mathbf{H}_{1}^{*} \\
\mathbf{H}_{3} & \mathbf{H}_{4} \\
-\mathbf{H}_{4}^{*} & \mathbf{H}_{3}^{*}
\end{array}\right]
$$

Computing $\mathbf{H}^{H} \mathbf{H}$, a $4 \times 4$ matrix is obtained similar as in the $4 \times 1$ antenna case, however with twice the diversity. Thus in this case a diversity of eight is achieved with a data rate of one.

On the other hand, by transmitting the sequences only twice, i.e. by truncating the Four Antenna Scheme in (7),

$$
\mathbf{S}_{\text {truncated }}=\left[\begin{array}{rrrr}
s_{1} & s_{2} & s_{3} & s_{4} \\
s_{2}^{*} & -s_{1}^{*} & s_{4}^{*} & -s_{3}^{*}
\end{array}\right],
$$

the received signals at the two antennas can be formed to

$$
\begin{aligned}
{\left[\begin{array}{l}
y_{11} \\
y_{12} \\
y_{21} \\
y_{22}
\end{array}\right] } & =\left[\begin{array}{rrrr}
h_{11} & h_{12} & h_{13} & h_{14} \\
-h_{12}^{*} & h_{11}^{*} & -h_{14}^{*} & h_{13}^{*} \\
h_{21} & h_{22} & h_{23} & h_{24} \\
-h_{22}^{*} & h_{21}^{*} & -h_{24}^{*} & h_{23}^{*}
\end{array}\right]\left[\begin{array}{l}
s_{1} \\
s_{2} \\
s_{3} \\
s_{4}
\end{array}\right] \\
& =\text { Hs. }
\end{aligned}
$$

Thus, computing $\mathbf{H}^{H} \mathbf{H}$ results simply in the following block matrix

$$
\mathbf{H}^{H} \mathbf{H}=\left[\begin{array}{rr}
\gamma_{1} \mathbf{I} & \mathbf{B} \\
\mathbf{B}^{H} & \gamma_{2} \mathbf{I}
\end{array}\right]
$$

with $\gamma_{1}=\left|h_{11}\right|^{2}+\left|h_{12}\right|^{2}+\left|h_{13}\right|^{2}+\left|h_{14}\right|^{2}$ and $\gamma_{2}=\left|h_{21}\right|^{2}+$ $\left|h_{22}\right|^{2}+\left|h_{23}\right|^{2}+\left|h_{24}\right|^{2}$. Due to the condition $\mathbf{B}^{H} \mathbf{B}=\mathbf{B B}^{H}$ such matrix can be inverted with a $2 \times 2$ matrix inversion rather than a $4 \times 4$.

$$
\left[\mathbf{H}^{H} \mathbf{H}\right]^{-1}=\left[\begin{array}{rr}
\gamma_{2} \mathbf{I} & -\mathbf{B} \\
-\mathbf{B}^{H} & \gamma_{1} \mathbf{I}
\end{array}\right]\left[\begin{array}{ll}
\mathbf{C} & \varnothing \\
\varnothing & \mathbf{C}
\end{array}\right]
$$

with $\mathbf{C}=\left[\gamma_{1} \gamma_{2} \mathbf{I}-\mathbf{B B}^{H}\right]^{-1}$. Thus, the underlying Alamouti scheme gives us the advantage of lower complexity while the BLAST scheme offers higher data rate. This mode was also proposed in [4] under the name Double Space-Time Transmit Diversity (D-STTD).

Simulation results for D-STTD in independent Rayleigh fading are shown in Fig.2. If compared to the low-rate scheme (7) (see also Figure 3 in [3]) it is observed that the $\mathrm{ML}$ receiver performs about $1 \mathrm{~dB}$ better at a BER of $10^{-3}$ while the ZF receiver performs worse by $4 \mathrm{~dB}$ when comparing to [3]. The difference between $\mathrm{ML}$ and $\mathrm{ZF}$ receiver performance is now much more pronounced.

Finally the third transmission mode would send only one set of four symbols to the four transmit antennas. The corresponding matrix $\mathbf{H}^{H} \mathbf{H}$ is not of full rank and therefore, cannot be inverted. The entries on its diagonal consist of two times diversity terms like $\left|h_{11}\right|^{2}+\left|h_{12}\right|^{2}$. The decoding can only be performed with an ML decoder[11] allowing only for diversity of two but with a data rate of four. 


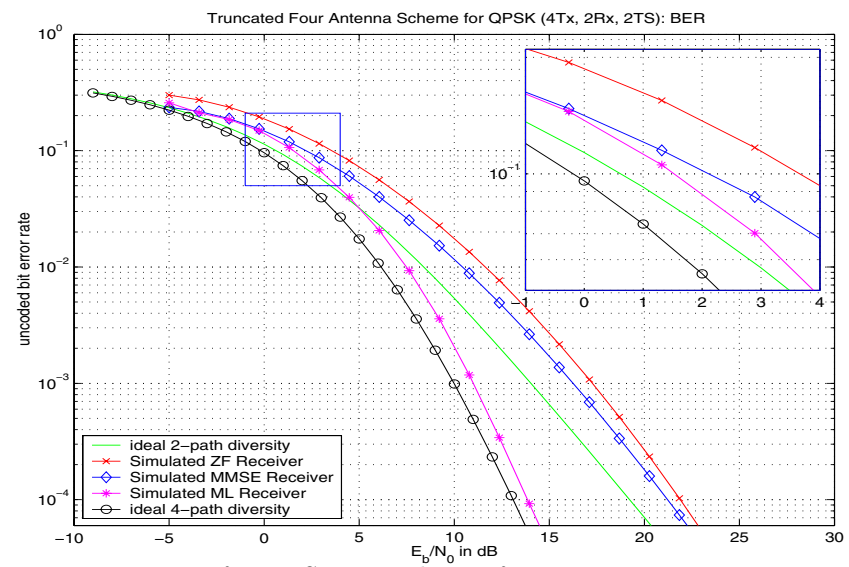

Fig. 2. BER for D-STTD scheme for various receiver types.

Example 4.2 Same is true for an eight by two scheme using the four antenna extended Alamouti scheme with a BLAST transmission achieving a transmission rate of two. Two sets of four symbols each are used to build the $4 \times$ 4 matrices $\mathbf{H}_{11}, \mathbf{H}_{12}, \mathbf{H}_{21}, \mathbf{H}_{22}$ for the four corresponding block transmissions. The transmission matrix $\mathbf{H}$ now reads

$$
\left[\begin{array}{ll}
\mathbf{H}_{11} & \mathbf{H}_{12} \\
\mathbf{H}_{21} & \mathbf{H}_{22}
\end{array}\right]
$$

and the term $\mathbf{H}^{H} \mathbf{H}$ is of the form

$$
\mathbf{H}^{H} \mathbf{H}=\left[\begin{array}{rr}
\alpha_{1} \mathbf{I}_{4}+\beta_{1} \mathbf{J}_{4} & \mathbf{B} \\
\mathbf{B}^{H} & \gamma_{2} \alpha_{2} \mathbf{I}_{4}+\beta_{2} \mathbf{J}_{4}
\end{array}\right]
$$

with

$$
\mathbf{J}_{4}=\left[\begin{array}{rr}
\varnothing & \mathbf{J}_{2} \\
-\mathbf{J}_{2} & \varnothing
\end{array}\right] .
$$

Thus, only a $4 \times 4$ matrix inverse is required. The remaining parts can be computed with the low complexity techniques as described before. Gaining such insight the following statement can be made.

Proposal 4.1 Given a transmission scheme with $M=2^{m}$ transmit and $N=2^{n}$ receive antennas, all possible diversity numbers (in multiples of $2^{k}$ ) ranging from one to $L=M N$ and data rates from one to $L$ can be achieved, as long the product of data rate and diversity number remains $L$.

On non-flat fading channels the UMTS transmission allows to increase the diversity by assigning a number of fingers to each major energy contribution. In this case, all finger values are combined in a correspondingly larger matrix $\mathbf{H}$. Assume that $K$ reflections at $K$ different delay times $\tau_{k}$ exist:

$$
\tilde{\mathbf{H}}=\sum_{k=1}^{K} \mathbf{H}_{k} \delta\left(t-\tau_{k}\right)
$$

When ordering the so received values block by block after another in one new matrix $\mathbf{H}$, the following is obtained

$$
\mathbf{H}=\left[\mathbf{H}_{1}, \mathbf{H}_{2}, \ldots, \mathbf{H}_{K}\right]
$$

However, $\mathbf{H}^{H} \mathbf{H}$ remains of the same size as before. The various fingers only contribute to (up to $\mathrm{K}$ times) higher diversity gain allowing to utilize BLAST schemes in which $\mathbf{H}^{H} \mathbf{H}$ would not be of full rank in a flat Rayleigh scenario.

\section{Concluding Remarks}

A very flexible and at the same time simple block coding scheme based on extended Alamouti schemes was presented. It was shown by many examples that such block coding schemes offer much flexibility at transmitter and receiver. Knowing the number of receiver antennas at the transmitter suffices for selecting the most suitable scheme out of a wide range of possible STC schemes allowing high flexibility in selecting data rate and diversity gain. This allows the service provider to select the optimal transmission scheme, for example in UMTS, individually selected for each supported mobile.

Furthermore, such extended Alamouti schemes can be described by very few values (in the simple case of a $4 \times 1$ scheme by only two real values $\left\{X, h^{2}\right\}$ ). Although random matrices, the transmission matrix $\mathbf{H}$ is now so well structured, that the eigenvectors are all known at the transmitter. Thus, simple feedback information of a few characteristic values from the receiver allows to support optimal transmission on eigenmodes[12].

\section{REFERENCES}

[1] V. Tarokh, N. Seshadri, A.R. Calderbank. Space-time codes for high data rate wireless communication: Performance criterion and code construction. IEEE Trans. Inf. Theor., 44(2):744-765, March 1998.

[2] S.M. Alamouti. A simple transmitter diversity scheme for wireless communications. IEEE J. Sel. Ar. Comm., 16(8):14511458, October 1998.

[3] M. Rupp, C.F. Mecklenbräuker. On Extended Alamouti Schemes for Space-Time Coding In WPMC'02, Honolulu, Oct. 2002.

[4] E.N. Onggosanusi, A.G. Dabak, T.M. Schmidl. High rate spacetime block coded scheme: performance and improvement in correlated fading. IEEE Wireless Communications and Networking Conference, March 2002.

[5] B.Hochwald, T.L.Marzetta, C.B.Papadias. A transmitter diversity scheme for wideband CDMA systems based on space-time spreading. IEEE J. Sel. Ar. Comm., 19(1):48-60, Jan. 2001.

[6] O. Tirkkonen, A. Boariu, A. Hottinen. Minimal nonorthogonality rate 1 space-time block code for $3+$ Tx antennas. In Proc. IEEE ISSSTA 2000, vol. 2, pp. 429-432, Sep. 2000.

[7] J.H. Winters, J. Salz, R.D. Gitlin. The impact of antenna diversity on the capacity of wireless communication systems. IEEE Trans. Comm., 42(2/3/4):1740-1750, February 1994.

[8] C.C. Martin, J.H. Winters, N.R. Sollenberger. MIMO radio channel measurements: performance comparison of antenna configurations. In 54 $4^{\text {th }}$ IEEE Vehicle Technology Conf. (VTC 2001 Fall), vol. 2, 1225-1229, Atlantic City, USA, October 2001.

[9] T.H. Liew, L. Hanzo. Space-time codes and concatenated channel codes for wireless communications. Proceedings of the IEEE, 90(2):187-219, February 2002.

[10] P.W. Wolniansky, G.J. Foschini, G.D. Golden, R.A. Valenzuela. V-BLAST: An architecture for achieving very high data rates over rich-scattering wireless channels. In Proc. Int. Symp. Signals, Systems, and Electronics (ISSSE-98), Pisa, Italy, 1998.

[11] R. van Nee, A. van Zelst, G. Awater. Maximum likelihood decoding in a space division multiplexing system. In $V T C^{\prime} 00$ Spring, $51^{\text {st }}$ IEEE Vehicular Technology Conference (VTC'OO Spring), 6-10, Tokyo, Japan, May 2000.

[12] G. Gritsch, H. Weinrichter. Adaptive subspace modulation in spatially correlated MIMO systems. In Proc. PIMRC, Lisboa, Portugal, September 2002. 